\title{
ON SOME HARDY-TYPE INEQUALITIES FOR GENERALIZED FRACTIONAL INTEGRALS
}

\author{
Muhammad Samraiz, ShafQAT Shahzadi, SaJid IQbal \\ AND ŽIVORAD TOMOVSKI
}

\begin{abstract}
In this article we establish the variant of Hardy-type and refined Hardy-type inequalities for a generalized Riemann-Liouville fractional integral operator and Riemann-Liouville $k$-fractional integral operator using convex and monotone convex functions. We also discuss one dimensional cases of our related results. As special cases of our general results we obtain the consequences of Iqbal et al. [11]. We also obtained exponentially convex linear functionals for the generalized fractional integral operators. Moreover, it includes Cauchy means for the above mentioned operators.
\end{abstract}

Mathematics subject classification (2010): 26D15, 26D10, 26A33, 34B27.

Keywords and phrases: Hardy-type inequalities, fractional integral operators, convex function, mean value theorem, exponential convexity.

\section{REFERENCES}

[1] S. Abramovich, K. Krulić, J. PeČarić, L. E. Persson, Some new refined Hardy type inequalities with general kernels and measures, Aequationes Mathematicae 79, 157-172, (2010).

[2] E. Adeleke, A. Čižmešija, J. Oguntuase, L. E. Persson, D. Pokaz, On a new class of Hardy-type inequalities, J. Inequal. Appl., (2012).

[3] S. N. Bernstein, Sur les fonctions absolument monotones, Acta Math. 52, 1-66, (1929).

[4] A. ČIŽMeŠIJA, K. KRulić, J. PeČArić, Some new refined Hardy-type inequalities with kernels, J. Math. Inequal. 4 (4), 481-503, (2010).

[5] A. ČIŽMEŠIJA, K. KRUlić, J. PEČARIĆ, A new class of general refined Hardy-type inequality with kernels, Rad HAZU, 17, 53-80, (2013).

[6] N. Elezović, K. Krulić, J. PeČArić, Bounds for Hardy type differences, Acta Mathematica Sinica, English Series 27 (4), 671-684, (2011).

[7] I. S. GRAdshteyn, I. M. RyZhiK, Table of Integrals, Series and products, Academics Press, Elsvier, (2007).

[8] S. IQbal, K. Krulić, J. PeČArić, On an inequality of H. G. Hardy, J. Inequal. Appl., Article ID 264347, (2010).

[9] S. IQbal, K. KRUlić, J. PeČARIĆ, On an inequality for convex function with some applications on fractional derivatives and fractional integrals, J. Math. Inequal. 5 (2), 219-230, (2011).

[10] S. IQbal, K. Krulić, J. PeČARIĆ, D. PoKaZ, n-Exponential Convexity of Hardy-type and Boastype functionals, J. Math. Inequal. 7, (4), 739-750, (2013).

[11] S. IQbAL, J. PeČARIĆ, M. SAMraiz, N. Sultana, Applications of Refined Hardy-type Inequalities, Math. Inequal. \& Appl. 18, (4), 1539-1560, (2015).

[12] S. Iqbal, J. PeČarić, M. Samraiz, Ž. Tomovski, On Some Hardy-type Inequalities for Fractional Calculus Operators, Banach J. Math. Anal. 11 (2), 438-457, (2017).

[13] S. Kaijser, L. Nikolova, L. E. Persson, A. Wedestig, Hardy-Type Inequalities via Convexity, Math. Inequal. \& Appl. 8 (3), 403-417, (2005).

[14] U. N. Katugampola, New approach to a generalized fractional integral, Appl. Math. Comput. 218, 860-865, (2011). 
[15] S. Kawashima, K. Kurata, Hardy type inequality and application to the stability of degenerate stationary waves, J. Funct. Anal. 257, 1-19, (1983).

[16] A. A. Kilbas, H. M. SRivastava, J. J. Trujillo, Theory and Applications of Fractional Differential Equations, North-Holland Mathematics Studies 204, Elsevier, New York-London, (2006).

[17] V. KokilashVili, A. Mes KhI, L. E. Person, Weighted norm inequalities for integral transformations with product kernels, Nova Science Publishers, Inc., New York, (2009).

[18] K. Krulić, J. PeČArić, L. E. Persson, Some new Hardy type inequalities with general kernels, Math. Inequal. \& Appl. 12 (3), 473-485, (2009).

[19] A. Kufner, L. E. Person, N. SAmko, Weighted Inequalities of Hardy-type, Second Edition, World Scientific, (2017).

[20] S. Mubeen, G. M. Habibullah, k-Fractional Integrals and Application, Int. J. Contemp. Math. Sciences 7 (2), 89-94, (2012).

[21] S. Mubeen, S. IQbaL, Grüss type integral inequalities for generalized Riemann-Liouville $k$ fractional integrals, J. Inequal. \& Appl. 109, (2016).

[22] C. Niculescu, L. E. Persson, Convex functions and their applications. A contemporary approach, CMC Books in Mathematics Springer, New York, (2006).

[23] B. Opic, A. Kufner, Hardy-type inequalities, Pitman Research Notes in Mathematics Series, Longman Scientific and Technical, Harlow, (1990).

[24] J. PEČARIĆ, J. Perić, Improvements of the Giaccardi and the Petrović inequality and related Stolarsky type means, Analysis of the University of Craiova, Mathematics and Computer Science Series. 39, (1), 65-75, (2012). 